\title{
Diagnostic biomarkers for USD via serum and urinary amino acid profiles using a metabolomics- based strategy
}

Yao Gao ( $\sim$ gaoyao21@163.com )

Fujian Medical University https://orcid.org/0000-0003-4722-5066

\section{Sisi Gong}

Fujian Medical University

Xinxin Lin

Fujian Medical University

Tianwen Zhang

Fujian Fishery Resources Monitoring Center

\section{Beiying Zhang}

Fujian Medical University

\section{Minjie Tang}

First Affiliated Hospital of Fujian Medical University

\section{Min Chen}

Fujian Medical University

\section{Qishui Ou}

First Affiliated Hospital of Fujian Medical University

Houping Mao

First Affiliated Hospital of Fujian Medical University

\section{Research article}

Keywords: amino acid profile, urinary stone disease, GC-MS, early diagnosis, metabolomics-based strategy

Posted Date: June 18th, 2021

DOI: https://doi.org/10.21203/rs.3.rs-629423/v1

License: (1) (1) This work is licensed under a Creative Commons Attribution 4.0 International License. Read Full License 


\section{Abstract}

Background: Urinary stone disease (USD) is a common disease with a high prevalence, a high recurrence rate and a lack of effective diagnostic biomarkers.

Methods: To explore the potential biomarkers for USD early diagnosis, the serum and urinary amino acid (AA) profiles of 74 USD patients were compared with 35 healthy controls $(\mathrm{HC})$ via gas chromatography coupled with mass spectrometry (GC-MS).

Results: Based on the serum and urinary AA profiles analyzed by metabolomic strategy, a group of 5 differentially expressed AAs including Ser, Glu, Asp, Ile and Gly and 7 metabolic pathway disorders in patients were revealed.

Conclusions: These serum and urinary AA profiles could provided clinical biomarkers for USD early diagnosis, and also indicated that some specific AA metabolic pathways might play important roles in the pathogenesis of USD.

\section{Background}

Urinary stone disease (USD) whose clinical manifestations are infection, flank pain, nausea, vomiting, ureteral obstruction and more seriously, renal failure [1], is a common disease with the characteristics of high prevalence and recurrence [2]. It is estimated that around 10\% of the USA residents were suffered from USD [3] and up to 2017, approximately one in 17 adults in China were affected by the disease [4]. Due to the lack of early prevention and adequate urological care, it is investigated that the recurrence rate is gradually increasing over time[5]. Recent studies have indicated that USD appears to be associated with multiple factors including dietary habits, natural environment and social living conditions [6], but the exact pathophysiological mechanism remains to be elucidated. Above all, early diagnosis and prompt treatment are particularly needed to slow disease progression and improve patients' quality of life.

Currently, imaging is the main clinical diagnostic method of USD including computed tomography (CT) and ultrasonography, while biochemistry testing in the clinical laboratory is also used to help assess the progression of the disease [7]. Nevertheless, imaging examinations are radioactive and expensive, even the clinical laboratory results can't evaluate disease comprehensively. There are still no reliable markers with high sensitivity and specificity that could allow for a quick diagnosis at an early stage. Hence, developing a more accurate, economic and radiation-free detection approach to signal the onset of USD timely is desirable and warranted.

AAs and their metabolites are the structural building blocks for the biosynthesis of peptides and proteins, playing crucial biological roles in living organisms [8]. The metabolism, as well as the concentration of AAs, could be influenced in pathological conditions, so AAs could be a key factor in relation to disease progression [9]. With the purpose of early diagnosis and pathogenesis exploration, AA analyses have been processed in aortic dissection, Parkinson's disease, type 2 diabetes mellitus and so on [9-11]. Duan 
et al have shown several metabolic biomarkers in urine samples may be related to USD based on nuclear magnetic resonance spectroscopy (NMR), such as creatinine, citrate, glycine (Gly), glutamine (GIn) and so on [12]. Atanassova et al have discovered that there was a remarkable difference in urinary amino acid (AA) concentrations between renal calculi patients and healthy controls $(\mathrm{HC})$ via high-performance liquid chromatography (LC), including serine (Ser), Gly, taurine and i-leucine [13]. Gao et al reported that in a trial conducted on a mouse model of renal calcium oxalate ( $\mathrm{CaOx}$ ) crystal deposition, 30 differently expressed urinary AAs including arginine (Arg), tyrosine (Tyr) and tryptophan (Try) were the putative markers of crystal-induced renal damage [14]. These evidences above manifested that AAs may be responsible for USD and there were AA metabolic pathways perturbations that occurred in patients. However, there is still insufficient research to study USD from the AA profiles in both serum and urine samples. Therefore, it is meaningful and promising to conduct serum and urinary AA profiling to seek the underlying biomarkers for USD early diagnosis.

Presently, the major technologies for AA profile analysis are NMR, LC coupled with mass spectrometry and gas chromatography coupled with mass spectrometry (GC-MS). With highly repeatable fragmentation of the compounds, GC-MS has many established databases which could support the identification process [15]. Till now, this well-established technique has been widely used in many disease studies such as myocardial infarction, atrial fibrillation and osteoarthritis [16-18]. Additionally, serum and urine were both regarded as the ideal biomedium because they are easily available and nonradiative. Besides that, they have a pool of metabolic information that can reflect the pathogenesis of disease more intuitively $[19,20]$. Along this line, GC-MS is suitable for AA profile detection in two sample types.

Here, our project was set out to explore the AA profiles of USD in serum and urine samples via a GC-MS platform using a metabolomics-based strategy. Screening potential predictors assisting imaging examinations for early diagnosis would be helpful to gain broader biological information of USD and be prompted to improve the living quality of patients in the future.

\section{Methods}

\subsection{Ethics}

The study was carried out in accordance with the Declaration of Helsinki. The collection activities of all biological samples were approved by the Ethics Committee of Fujian Medical University ([2014] No. 106), while an oral health questionnaire and written informed consent was acquired from all participants.

\subsection{Clinical samples collection}

To establish the comprehensive AA profiles of USD, independent serum and urine samples were collected from the First Affiliated Hospital of Fujian Medical University during the 2015-2017 period. A total of 109 subjects were enrolled in this work including 74 USD patients and $35 \mathrm{HC}$. All patients were diagnosed by CT or ultrasonography at the Department of urology according to the European Association of Urology guideline [21] and met the inclusion criteria (first-episode, without surgical treatment). Exclusion criteria were pregnancy, cancer, diabetes, hypertension and renal dysfunction as well as any other diseases which 
might be associated with AA disorders. The control group consisted of healthy volunteers with normal results in physical check-ups and laboratory examinations. The HC group matched the USD group in terms of age and gender. About $80 \%$ of samples from each group were randomly assigned as the training set ( 59 patients and $28 \mathrm{HC}$ ), and the remaining samples were classified as the test set ( 15 patients and 7 HC). Characteristics of the two groups were presented in Table 1.

Fasting blood samples from the antecubital vein were collected into gel-based separation blood collection tubes $(2 \mathrm{~mL})$ and the first-morning midstream urine samples were collected into sterile tubes $(10 \mathrm{~mL})$. To prevent degradation, they were centrifuged at $1,000 \mathrm{~g}$ for $10 \mathrm{~min}$ within 1 hour. Aliquots of the supernatants were stored at $-80^{\circ} \mathrm{C}$ until the GC-MS analysis was conducted.

Table 1

Basic information of participants involved in the study.

\begin{tabular}{|lllll|}
\hline & Training set & \multicolumn{3}{l|}{ Test set } \\
\hline Group & USD & HC & USD & HC \\
\hline Subjects,N & 59 & 28 & 15 & 7 \\
\hline Age (years) & $46.42 \pm 13.72$ & $43.66 \pm 12.59$ & $46.85 \pm 13.32$ & $43.26 \pm 13.49$ \\
\hline Gender (male/female) & $33 / 26$ & $16 / 12$ & $8 / 7$ & $4 / 3$ \\
\hline
\end{tabular}

\subsection{Instruments and reagents}

An Agilent 5977A-7890B GC-MS instrument and $2.0 \mathrm{~mL}$ vials were purchased from Agilent (Palo Alto, USA); ultrapure water was produced by a Milli-Q water purification system (Millipore, USA); the BP 211 D analytical balance was employed from Sartorius (Gottingen, Germany); 3-30k high-speed centrifuge was obtained from Sigma-Aldrich (St. Louis, MO).

The mixed standard solution of 17 AAs $(2500 \mu \mathrm{mol} / \mathrm{L}$, concentration of cystine (CySS) was $1250 \mu \mathrm{mol} / \mathrm{L}$ ) was purchased from Sigma-Aldrich (St. Louis, MO) containing alanine (Ala), Arg, aspartic acid (Asp), CySS, glutamic acid (Glu), lysine (Lys), leucine (Leu), Ser, threonine (Thr), Tyr, valine (Val), histidine (His), isoleucine (Ile), methionine (Met), Gly, phenylalanine (Phe) and proline (Pro). Try, cysteine (Cys), Gln and four isotope-labeled amino acids including ${ }^{13} \mathrm{C}_{3}$-Ala, ${ }^{13} \mathrm{C}_{3}$-Ser, ${ }^{13} \mathrm{C}_{6}$-Tyr, and ${ }^{13} \mathrm{C}_{6}$-Phe were supplied by Toronto Research Chemicals (North York, ON, Canada). LC-grade acetonitrile, anhydrous ethanol and isooctane were obtained from Merck (Darmstadt, Germany). Analytical reagent (AR) grade L-2chlorophenyl alanine (internal standard, IS), hydrochloric acid and sodium hydroxide were acquired from Shanghai Sinopharm Chemical Reagent Co., Ltd (Shanghai, China). Pyridine (purity 99.8\%) was purchased from Shanghai Aladdin Bio-Chem Technology Co. Ltd (Shanghai, China). Isobutyl chloroformate (IBCF, AR grade) was purchased from Shanghai ANPEL Scientific Instruments Co. Ltd (Shanghai, China). 


\subsection{Standard solution preparation}

Firstly, a pooled stock solution ( $2000 \mu \mathrm{mol} / \mathrm{L})$ consisting of Try, Cys and GIn was prepared in hydrochloric acid $(0.1 \mathrm{~mol} / \mathrm{L})$. Then, the mixed 20 AAs stock standard solution $(500 \mu \mathrm{mol} / \mathrm{L}$, concentrations of CySS was $250 \mu \mathrm{mol} / \mathrm{L})$ was prepared by diluting the above pooled stock solution $(2000 \mu \mathrm{mol} / \mathrm{L})$ and $17 \mathrm{AAs}$ standard solution $(2500 \mu \mathrm{mol} / \mathrm{L}$, concentration of CySS was $1250 \mu \mathrm{mol} / \mathrm{L})$ with hydrochloric acid $(0.1$ $\mathrm{mol} / \mathrm{L})$.

Subsequently, a series of standard operating solutions containing 20 AAs at the final concentrations of 1 , $5,10,25,50,100$ and $250 \mu \mathrm{mol} / \mathrm{L}$ (the concentrations of CySS were $0.5,2.5,5,12.5,25,50$ and 125 $\mu \mathrm{mol} / \mathrm{L})$ were prepared by diluting the mixed 20 AAs stock standard solution with hydrochloric acid $(0.1$ $\mathrm{mol} / \mathrm{L}$ ). The concentrations of four isotope-labeled AAs for recovery experiments were $500 \mu \mathrm{mol} / \mathrm{L}$. Finally, all of the solutions were stored at $4{ }^{\circ} \mathrm{C}$ within one week until use.

\subsection{Serum samples pretreatment}

Each serum sample was thawed at room temperature for 30 min and immediately centrifuged at 1,000 g $4^{\circ} \mathrm{C}$ for $5 \mathrm{~min}$. AAs were extracted from a $100 \mu \mathrm{L}$ aliquot of serum supernatant by addition of $400 \mu \mathrm{L}$ of acetonitrile and $200 \mu \mathrm{L}$ of ethyl alcohol in a $2.0 \mathrm{~mL}$ centrifuge tube. The tube was swirled for $2 \mathrm{~min}$ and rest at $4^{\circ} \mathrm{C}$ for $20 \mathrm{~min}$ followed by centrifuging at $1,000 \mathrm{~g} 4^{\circ} \mathrm{C}$ for $5 \mathrm{~min}$. Then, a $500 \mu \mathrm{L}$ of supernatant was transferred to a $2.0 \mathrm{~mL}$ vial and subsequently mixed with $100 \mu \mathrm{L}$ of IS solution ( $L$-2-chlorophenyl alanine in ultrapure water, $0.1 \mathrm{mg} / \mathrm{mL}$ ), $400 \mu \mathrm{L}$ of ultrapure water and $10 \mu \mathrm{L}$ of sodium hydroxide solution (sodium hydroxide in ultrapure water, $7 \mathrm{~mol} / \mathrm{L}$ ). The mixture was blended for $1 \mathrm{~min}$ and then mixed with $50 \mu \mathrm{L}$ of pyridine followed by a 1 min vortex. Afterwards, the solution was mixed with $40 \mu \mathrm{L}$ of IBCF[22] and $300 \mu \mathrm{L}$ of isooctane for derivatization. Meanwhile, the vial was rest and deflated for $5 \mathrm{~min}$. Finally, $100 \mu \mathrm{L}$ of the derivatized supernatant was transferred into the vial for GC-MS analysis.

\subsection{Urinary samples pretreatment}

All urinary samples were thawed at room temperature and centrifuged at 1,000 $\mathrm{g}$ for $5 \mathrm{~min}$ before pretreatment. In a $2.0 \mathrm{~mL}$ vial, $100 \mu \mathrm{L}$ of urine supernatant was mixed with $100 \mu \mathrm{L}$ of ultrapure water and $0.1 \mathrm{mg} / \mathrm{mL}$ IS solution. Then the metabolites were extracted with $400 \mu \mathrm{L}$ of anhydrous ethanol. In order to alkalinize the solutions and catalyze the reaction, $100 \mu \mathrm{L}$ of $7 \mathrm{~mol} / \mathrm{L}$ sodium hydroxide solution and 100 $\mu \mathrm{L}$ of pyridine were successively placed into the vial. After mixing with $50 \mu \mathrm{L}$ of IBCF [22], the vial was well shaken and the liberated carbon dioxide was escaped from the vial for about $5 \mathrm{~min}$. Subsequently, the solution was mixed with $300 \mu \mathrm{L}$ of isooctane followed by swirling and resting for about $10 \mathrm{~min}$. Lastly, $100 \mu \mathrm{L}$ of the derivatized supernatant was collected for further GC-MS analysis. In addition, the creatinine concentration was determined by Jaffe's reaction.

\subsection{GC-MS conditions}

Our study was performed on an Agilent 7890B-5977A GC-MS system (Agilent, USA). One $\mu$ L aliquot of the derivatized solution was injected into an HP-5MS capillary column $(30 \mathrm{~m} \times 0.25 \mathrm{~mm} \times 0.25 \mu \mathrm{m}$, Agilent, USA). High-purity helium (99.9996\%, Linde, China) was used as the carrier gas at a constant flow rate of 1 $\mathrm{mL} / \mathrm{min}$. The temperature of the injection port was set at $260^{\circ} \mathrm{C}$ and the $\mathrm{GC}$ oven temperature was 
programmed as follows: an initial temperature was set at $80^{\circ} \mathrm{C}$ for $2 \mathrm{~min}$, then ramped to $140^{\circ} \mathrm{C}$ by $10^{\circ} \mathrm{C} / \mathrm{min}$, then to $240^{\circ} \mathrm{C}$ by $4^{\circ} \mathrm{C} / \mathrm{min}$, and then to $280^{\circ} \mathrm{C}$ by $10^{\circ} \mathrm{C} / \mathrm{min}$ and maintained for $3 \mathrm{~min}$. The energy was set at $70 \mathrm{eV}$ in electron impact ionization, with selected ion monitoring (SIM) mode to acquire the data of target AAs. The temperature of transfer interface, ion source and quadrupole were set at 260, 230 and $150^{\circ} \mathrm{C}$ respectively with a solvent delay of $9.5 \mathrm{~min}$. All these parameters were optimized to obtain high mass accuracy and reproducibility.

\subsection{Data Analysis}

Data pretreatment: Peak alignment, background noise subtraction and data reduction were processed by ChemStation software (Agilent) during the initial step in an automated and unbiased way. Then, each peak area on the chromatograms of serum samples was standardized with that of IS. Additionally, the peak area of urinary samples was further standardized with that of IS and creatinine.

Multivariate pattern recognition techniques: Based on the training set, principal component analysis (PCA) and orthogonal partial least squares-discriminant analysis (OPLS-DA) with the Pareto scaling technique were carried out using SIMCA-P v14.0 software (Umetrics AB, Sweden). PCA is an unsupervised multivariate analysis to acquire an overview of the data. OPLS-DA, a kind of supervised multivariate statistical analysis method, can visualize the distinction between two groups. And the quality of the models was assessed by the parameters $R^{2} X, R^{2} Y$ and $Q . R^{2} X$ and $R^{2} Y$ were used to explain the percentages of $x$-variables, $y$-variables of the dataset in the model, respectively. $Q^{2}$ represents the capacity of the predictive performance of the models. The AAs with importance in the projection (VIP) value greater than 1.0 from the loading plots of OPLS-DA were considered to be likely responsible for differentiating groups[23]. In order to evaluate the validity of the model and guard against over-fitting, rigorous permutation testing $(n=100)$ were performed. The test set was performed to verify the predictive ability of the established OPLS-DA models.

Univariate method: The independent-samples t-test[24] was performed by SPSS Statistics 17.0 (IBM Corp., USA) to assess the statistical differences of AAs between USD and HC groups. The latent AA markers were further extracted based on the critical $p$-value set to 0.05 .

Diagnostic ability analyses: Diagnostic property of the latent markers was deduced by receiver operating characteristic $(\mathrm{ROC})$ curve analysis and binary logistic regression analysis. The optimal area under the curve (AUC), sensitivity and specificity were determined by the maximum of the Youden index. These analyses were also executed by SPSS 17.0 software.

Metabolic pathway analyses: Two web-based tools, MetaboAnalyst and Kyoto Encyclopedia of Genes and Genomes (KEGG), were employed to discover the disordered metabolic pathways.

\section{Results And Discussion}

\subsection{Optimization of biosamples pretreatment process}


In this study, the sample pretreatment processes for serum and urine were optimized in terms of protein precipitants, derivatization reagents and extractants. Firstly, different combinations and proportions of methanol, ethanol and acetonitrile were investigated as protein precipitants using an orthogonal experiment design. Best precipitation efficiency in serum was observed when using acetonitrile-ethanol $(2: 1 \mathrm{v} / \mathrm{v})$. However, ethanol was verified as the suitable precipitant for urine samples. Then, three derivatization reagents including trifluoroacetic anhydride, ethyl chloroformate, propyl chloroformate and IBCF were compared. The results indicated that when IBCF was performed as a derivatization reagent, the reaction time was short and the products were stable. Finally, the abilities of chloroform, n-hexane and isooctane to extract esterified AAs were compared. The results suggested that isooctane could be the optimum composition of solvents as the extraction buffer.

\subsection{GC-MS spectra of AAs from stock standard solutions}

GC-MS conditions were optimized based on the derivatized stock standard solution. In this work, the $80 \%$ rule was conducted to remove the missing values [25]. Moreover, limited by instrumental limits of detection (LODs) and derivation effects, a total of 16 AAs from the derivatized stock standard solution ( $50 \mu \mathrm{mol} / \mathrm{L}$, concentration of CySS was $25 \mu \mathrm{mol} / \mathrm{L}$ ) was determined. The total ion chromatogram (TIC) was presented in Supplemental Fig.S1, showing a strong signal and satisfactory separation. Meanwhile, the representative mass spectrums of several AAs, such as lle, Ser and Asp, were also depicted. Additionally, serum and urinary samples were analyzed separately in random order. As the natural abundance differences of AAs between the two types of samples, 14 serum AAs (Ala, Gly, Val, Leu, Ile, Pro, Ser, Asp, Met, Glu, Phe, Lys, Try, and Tyr) and 14 urinary AAs (Ala, Gly, Val, Leu, Ile, Pro, Ser, Asp, Met, Cys, Glu, Phe, Lys, and Try) were determined, respectively.

\subsection{Identification and quantitation}

A series of standard operating solutions, were prepared and derivatized as biosamples and immediately analyzed by GC-MS. And the target AAs were identified by retention time (RT) and the corresponding characteristic ions. The calibration curves were acquired in quantitative analyses, where the correlation coefficients $\left(R^{2}\right)$ were all greater than 0.9985 . In addition, the LODs of target AAs, based on a signal-tonoise ratio $(S / M)$ of 3 , were ranged from 0.1 to $4.0 \mu \mathrm{mol} / \mathrm{L}$. Regression equations, $R^{2}$, LODs and characteristic ions of 16 AAs were shown in Supplemental Table S1. The method was verified according to FDA guideline[26] on bioanalytical method validation and showed satisfactory compliance. Average recoveries were calculated by the use of spiking four isotope-labeled amino acids into random serum and urinary samples. They were $99.1 \%, 92.2 \%, 100.5 \%$, and $88.1 \%$ for ${ }^{13} \mathrm{C}_{3}-\mathrm{Ala},{ }^{13} \mathrm{C}_{3}-\mathrm{Ser}$, ${ }^{13} \mathrm{C}_{6}$-Tyr, and ${ }^{13} \mathrm{C}_{6}{ }^{-}$ Phe, respectively.

Therefore, these results indicated that this method was proved to be credible and robust for routine AA analysis.

\subsection{Serum AA profile and biomarkers selection}

An unsupervised PCA method based on serum samples was initially conducted to generate an unbiased overview of the difference in the AA profile between USD and HC groups. As shown in the score plot of 
PCA (Fig. 1a), in the training set, there was a separating tendency between the two groups. Then, a supervised OPLS-DA model was further established in order to maximize the separation and seek for the differential AAs. Figure $1 \mathrm{~b}$ and Supplemental Table S2 also displayed that samples of USD and HC formed two distinct groups, indicating certain AAs altered in USD patients. To validate the feasibility and reliability of the OPLS-DA model, permutation tests were conducted by repeating 100 times. In the crossvalidation plot (Fig. 1C), there was non-overfitting in this model as permuted $R^{2}$ and $Q^{2}$ values were lower than the original points to the right, and the $Q^{2}$ intercept was below zero [27]. The OPLS-DA model was also validated with a test set and proved to be properly designed to predict the classification.

The serum markers of AAs were selected according to the VIP value greater than 1.0 and $p<0.05$. Ultimately, 4 candidates in serum were selected, including Ser, Glu, Asp and Ile (Table 2). The relative concentrations of these potential serum biomarkers between USD and HC groups are shown in Fig. 2, where all of them had relatively lower concentrations in the USD group.

Herein, the serum Ser was obviously decreased in patients compared with those in healthy controls. And notably, the serum Ser displayed excellent effectiveness to identify the disease as its AUC value reached 0.844 , with a sensitivity, specificity and Youden index of $100.0 \%, 62.7 \%$, and 0.627 , respectively, where the ideal sensitivity may result from the limitation of sample capacity. This result implied that the serum Ser could become a positive signaling regulator for quick USD screening more convincingly.

Table 2

Significantly differential AAs in serum and urinary samples between USD and HC groups.

\begin{tabular}{|c|c|c|c|c|c|c|c|c|c|}
\hline \multirow[t]{2}{*}{ AA } & \multicolumn{4}{|c|}{ Serum samples } & \multicolumn{4}{|c|}{ Urinary samples } & \multirow[t]{2}{*}{ Pathway* } \\
\hline & VIP & $\begin{array}{l}p- \\
\text { value }\end{array}$ & FC & AUC & VIP & $\begin{array}{l}p- \\
\text { value }\end{array}$ & FC & AUC & \\
\hline Ser & 2.06 & $\begin{array}{l}1.68 \mathrm{E}- \\
07\end{array}$ & 0.77 & 0.844 & / & / & 1.16 & / & $\begin{array}{l}\text { Glycine, serine and } \\
\text { threonine metabolism }\end{array}$ \\
\hline Glu & 1.65 & $\begin{array}{l}1.46 \mathrm{E}- \\
03\end{array}$ & 0.69 & 0.738 & / & / & 1.30 & / & $\begin{array}{l}\text { D-Glutamine and D- } \\
\text { glutamate metabolism }\end{array}$ \\
\hline Asp & 1.19 & $\begin{array}{l}3.24 \mathrm{E}- \\
03\end{array}$ & 0.83 & 0.722 & 1.84 & $\begin{array}{l}6.90 \mathrm{E}- \\
04\end{array}$ & 1.59 & 0.683 & $\begin{array}{l}\text { Alanine, aspartate and } \\
\text { glutamate metabolism }\end{array}$ \\
\hline Ile & 1.04 & $\begin{array}{l}1.73 \mathrm{E}- \\
02\end{array}$ & 0.87 & 0.662 & l & $\begin{array}{l}1.04 \mathrm{E}- \\
03\end{array}$ & 1.43 & / & $\begin{array}{l}\text { Valine, leucine and } \\
\text { isoleucine biosynthesis }\end{array}$ \\
\hline Gly & l & / & 0.91 & / & 2.60 & $\begin{array}{l}2.90 \mathrm{E}- \\
02\end{array}$ & 0.80 & 0.643 & $\begin{array}{l}\text { Glycine, serine and } \\
\text { threonine metabolism }\end{array}$ \\
\hline
\end{tabular}

VIP was obtained from the OPLS-DA model with a threshold of 1.0;

$p$-value was calculated from $t$-test and selected with the criteria of 0.05 ; 
Fold change (FC) was the relative levels of each AAs in USD samples compared with HC samples;

AUC was calculated from ROC analysis and picked out when AUC was greater than 0.6;

* Metabolic pathway was suggested by MetaboAnalyst.

\subsection{Urinary AA profile and biomarkers selection}

The urinary data were also analyzed by PCA and OPLS-DA. It can be first observed that, in the training set, USD and HC groups had slight separation as exhibited in the PCA score plot (Fig. 1e). OPLS-DA model was subsequently carried out to highlight the difference of the endogenous AAs accounting for the grouping trends. As shown in Fig. $1 \mathrm{f}$ and Supplemental Table S2, the AA profile of patients was basically separated from that of $\mathrm{HC}$ obviously. Besides that, the result of the permutation test (Fig. $1 \mathrm{~g}$ ) indicated the feasibility of this model as no over-fitting existed.

The significantly expressed urinary AAs were mined with the same criteria and Gly and Asp were ultimately picked up as underlying biomarkers contributing to distinguish patients from healthy subjects (Table 2). As illustrated in Fig. 2, compared with HC, the urinary Gly level of USD patients was downregulated, while the concentration of Asp was increased in USD patients.

\subsection{Model verification}

In an attempt to assess the predictive abilities of the established OPLS-DA models, the serum and urinary test sets were applied, respectively. The predicted score plots of serum and urine were presented in Fig. 1d and Fig. 1h, respectively, where 15 USD patients and 7 healthy controls were correctly assigned. Hence, the OPLS-DA models of serum and urinary AA profiles indicated powerful predictability for USD.

\subsection{USD-related metabolic pathway analyses}

To explore possible pathways affected by USD, the above 5 differential expressed metabolites containing Ser, Glu, Asp, lle and Gly in serum and urine were pooled together for metabolic pathway analysis by MetaboAnalyst and KEGG database. A total of 20 pathways related to the 5 differential AAs was obtained as listed in Supplemental Table S3. And Supplemental Fig.S2 was plotted using the pathway impact as the abscissa and the $-\log (p)$ as the ordinate, where the circles represent metabolic pathways. The metabolic pathways which had greater importance were farther away from the origin. On the basis of $p$ value $>0.05$ and pathway-impact value $>0.1$, the results highlighted 7 highly influential metabolic pathways which were closely associated with USD, including aminoacyl-tRNA biosynthesis; glyoxylate and dicarboxylate metabolism; arginine biosynthesis; glutathione metabolism; alanine, aspartate and glutamate metabolism; glycine, serine and threonine metabolism; and D-glutamine and D-glutamate metabolism.

According to the metabolic pathway analyses above, the hypothesized USD-related pathways were shown in Fig. 3, which revealed that USD stone formation may be closely associated with these 
differential AAs and relevant metabolic pathways in USD. Glu, the vital intermediate of D-glutamine and D-glutamate metabolism, could be synthesized from a-ketoglutarate via amination to participate in the TCA cycle [9]. And as the important molecular integrator of alanine, aspartate and glutamate metabolism, Asp also could form oxaloacetate through transamination to involve in the TCA cycle. Besides, lle could generate acetyl-CoA indirectly to particularly involve in this energy metabolism [28], as well as valine, leucine and isoleucine biosynthesis. Gly could convert into Ser through serine hydroxymethyltransferase and vice versa to participate in the glycine, serine and threonine metabolism and glycolysis metabolism.

\subsection{ROC analysis for validation of potential biomarkers}

In order to investigate the diagnostic utility of the selected markers in clinical practice, ROC curve analysis was employed. The AUC manifested the diagnostic performance via assessing the sensitivity and specificity of the variables. Table 2 showed the AUC value of each putative marker in serum and urinary samples, indicating favorable diagnostic ability as all of them were greater than 0.6 and could become promising diagnostic biomarkers for early detection of USD.

Furthermore, in an attempt to improve the diagnostic accuracy of USD, serum Ser, Glu, Asp, lle and urinary Gly and Asp were combined into an integrated variable. ROC analysis with binary logistic regression was thereafter applied. Figure 4 presented the satisfactory performance of the integrated multi-markers panel, demonstrating a greater diagnosability to distinguish the patients from healthy people with a satisfactory AUC value of 0.890 , a global sensitivity of $78.0 \%$, specificity of $96.4 \%$ and Youden index of 0.744 . It should be noted that the integrated panel yielded a higher efficiency to diagnose than that of each amino acid for USD. This integrated panel might be an excellent performing indicator for USD and this AA profile analysis could be used in laboratory medicine in the future.

Our study has some limitations. The sample capacity is relatively small because we were limited to available serum and urine samples under strict condition which were concerned about medication history, underlying diseases and other factors.

\section{Conclusions}

In our study, a robust, extended and valid AA profile via a GC-MS platform has been successfully established using a metabolomics-based strategy. The project was designed with two types of samples including serum and urine to search for the latent and comprehensive biomarkers of USD. Overall, serum Ser, Glu, Asp, lle and urinary Asp and Gly were significantly altered in USD, implying energy homeostasis associated with AAs is disrupted in patients. These results provided some new useful penetration points which could be considered as potential biomarkers to distinguish patients, implying that serum and urinary AA profiling is a promising strategy for detecting specific biomarkers for USD. Serum Ser could be a sensitive individual indicator for USD screening. Meanwhile, a group of serum Ser, Glu, Asp, lle and urinary Asp and Gly would be treated as multi-markers for USD early diagnosis and used for metabolic pathway analyses and cytological study in order to excavate more underlying information associated with disease progression in the future. 


\section{Abbreviations}

USD, Urinary stone disease; GC-MS, gas chromatography coupled with mass spectrometry; PCA, principal component analysis; OPLS-DA, orthogonal partial least squares-discriminant analysis; AA, amino acid; CT, computed tomography; $\mathrm{CaOx}$, calcium oxalate; NMR, nuclear magnetic resonance spectroscopy; $\mathrm{HC}$, healthy controls; Ala, alanine; Arg, arginine; Asp, aspartic acid; CySS, cystine; Glu, glutamate; Lys, lysine; Leu, leucine; Ser, serine; Thr, threonine; Tyr, tyrosine; Val, valine; His, histidine; lle, isoleucine; Met, methionine; Gly, glycine; Phe, phenylalanine; Pro, proline; Try, tryptophan; Cys, cysteine; GIn, glutamine; AR, analytical reagent; IBCF, isobutyl chloroformate; SIM, selected ion monitoring; VIP, importance in the projection; ROC, receiver operating characteristic; AUC, area under the curve; KEGG, Kyoto Encyclopedia of Genes and Genomes; LODs, limits of detection; TIC, total ion chromatogram; RT, retention time; $\mathrm{R}^{2}$, correlation coefficient; $S / N$, signal-to-noise ratio; FC, fold change; TCA, tricarboxylic acid.

\section{Declarations}

Ethics approval and consent to participate The study was carried out in accordance with the Declaration of Helsinki. The collection activities of all biological samples were approved by the Ethics Committee of Fujian Medical University ([2014] No. 106), while an oral health questionnaire and written informed consent was acquired from all participants.

Consent for publication Written consent was obtained from study participants for publication.

Availability of data and material The datasets used during the current study are available from the corresponding authors on reasonable request.

Competing interests The authors declare no competing interests associated with the manuscript.

Funding This work was supported by National Natural Science Foundation of China [grant number 21405017], Fujian Natural Science Foundation [grant number 2018J01676], and College Students' Innovation and Entrepreneurship Training Program of Fujian Province [grant number 201610392079].

Authors' contributions Y.G., T.W.Z. and M.C. developed the study design. Y.G., T.W.Z., S.S.G. and B.Y.Z. conducted the GC-MS experiments. Y.G., X.X.L. and S.S.G. did the statistical analysis. M.J.T., Q.S.O. and H.P.M supplied the samples. Y.G. and B.Y.Z. collected the samples. Y.G., X.X.L., and S.S.G wrote the paper. M.C. and H.P.M explained the clinical results.

Acknowledgements We thank the USD and $\mathrm{HC}$ volunteers for their participation in sample donation for this study.

\section{References}

1. Yang $Y$, Deng $Y$, Wang Y: Major geogenic factors controlling geographical clustering of urolithiasis in China. Science of The Total Environment 2016, 571:1164-1171. 

oxalate urolithiasis in adults based on UPLC-Q-TOF/MS. J Chromatogr B Analyt Technol Biomed Life Sci 2019, 1124:290-297.

3. Ramaswamy K, Killilea DW, Kapahi P, Kahn AJ, Chi T, Stoller ML: The elementome of calciumbased urinary stones and its role in urolithiasis. Nat Rev Urol 2015, 12(10):543-557.

4. Zeng G, Mai Z, Xia S, Wang Z, Zhang K, Wang L, Long Y, Ma J, Li Y, Wan SP et al: Prevalence of kidney stones in China: an ultrasonography based cross-sectional study. Bju International 2017, 120(1):109-116.

5. Rule AD, Lieske JC, Li X, Melton LJ, 3rd, Krambeck AE, Bergstralh EJ: The ROKS nomogram for predicting a second symptomatic stone episode. Journal of The American Society of Neprology 2014, 25(12):2878-2886.

6. Lin SY, Lin CL, Chang CH, Wu HC, Chen WC, Wang IK, Liu YL, Sung FC, Chang YJ, Kao CH: Comparative risk of chronic kidney diseases in patients with urolithiasis and urological interventions: a longitudinal population-based study. Urolithiasis 2017, 45(5):465-472.

7. Scales CD, Jr., Tasian GE, Schwaderer AL, Goldfarb DS, Star RA, Kirkali Z: Urinary Stone Disease: Advancing Knowledge, Patient Care, and Population Health. Clinical Journal of the American Society of Nephrology 2016, 11(7):1305-1312.

8. Hanff E, Ruben S, Kreuzer M, Bollenbach A, Kayacelebi AA, Das AM, von Versen-Hoynck F, von Kaisenberg C, Haffner D, Uckert S et al: Development and validation of GC-MS methods for the comprehensive analysis of amino acids in plasma and urine and applications to the HELLP syndrome and pediatric kidney transplantation: evidence of altered methylation, transamidination, and arginase activity. Amino Acids 2019, 51(3):529-547.

9. Wang L, Liu S, Yang W, Yu H, Zhang L, Ma P, Wu P, Li X, Cho K, Xue S et al: Plasma Amino Acid Profile in Patients with Aortic Dissection. Sci Rep 2017, 7:40146.

10. Figura M, Kusmierska K, Bucior E, Szlufik S, Koziorowski D, Jamrozik Z, Janik P: Serum amino acid profile in patients with Parkinson's disease. PLOS One 2018, 13(1):e0191670.

11. Saleem T, Dahpy M, Ezzat G, Abdelrahman G, Abdel-Aziz E, Farghaly R: The Profile of Plasma Free Amino Acids in Type 2 Diabetes Mellitus with Insulin Resistance: Association with Microalbuminuria and Macroalbuminuria. Applied Biochemistry And Biotechnology 2019, 188(3):854-867.

12. Duan X, Zhang T, Ou L, Kong Z, Wu W, Zeng G: H NMR-based metabolomic study of metabolic profiling for the urine of kidney stone patients. Urolithiasis 2020, 48(1):27-35.

13. Atanassova SS, Panchev P, Ivanova M: Plasma levels and urinary excretion of amino acids by subjects with renal calculi. Amino Acids 2010, 38(5):1277-1282. 
14. Gao S, Chen W, Peng Z, Li N, Su L, Lv D, Li L, Lin Q, Dong X, Guo Z et al: Urinary metabonomics elucidate the therapeutic mechanism of Orthosiphon stamineus in mouse crystal-induced kidney injury. $J$ Ethnopharmacol 2015, 166:323-332.

15. Beccaria M, Franchina FA, Nasir M, Mellors T, Hill JE, Purcaro G: Investigation of mycobacteria fatty acid profile using different ionization energies in GC-MS. Analytical And Bioanalytical Chemistry 2018, 410(30):7987-7996.

16. Wang X, Wang D, Wu J, Yu X, Lv J, Kong J, Zhu G, Su R: Metabolic Characterization of Myocardial Infarction Using GC-MS-Based Tissue Metabolomics. Int Heart J 2017, 58(3):441-446.

17. She J, Guo M, Li H, Liu J, Liang X, Liu P, Zhou B, Liu S, Deng Y, Lou B et al: Targeting amino acids metabolic profile to identify novel metabolic characteristics in atrial fibrillation. Clin Sci (Lond) 2018, 132(19):2135-2146.

18. Carlson AK, Rawle RA, Wallace CW, Brooks EG, Adams E, Greenwood MC, Olmer M, Lotz MK, Bothner B, June RK: Characterization of synovial fluid metabolomic phenotypes of cartilage morphological changes associated with osteoarthritis. Osteoarthritis And Cartilage 2019, 27(8):11741184.

19. Yang XY, Zhang SN, Li XZ, Wang Y, Yin XD: Analysis of human serum metabolome for potential biomarkers identification of erosive oral lichen planus. Clin Chim Acta 2017, 468:46-50.

20. Osman D, Ali O, Obada M, El-Mezayen H, El-Said H: Chromatographic determination of some biomarkers of liver cirrhosis and hepatocellular carcinoma in Egyptian patients. Biomedical Chromatography 2017, 31(6).

21. Turk C, Petrik A, Sarica K, Seitz C, Skolarikos A, Straub M, Knoll T: EAU Guidelines on Interventional Treatment for Urolithiasis. European Urology 2016, 69(3):475-482.

22. Deng C, Li N, Zhang X: Rapid determination of amino acids in neonatal blood samples based on derivatization with isobutyl chloroformate followed by solid-phase microextraction and gas chromatography/mass spectrometry. Rapid Commun Mass Spectrom 2004, 18(21):2558-2564.

23. Tan B, Qiu Y, Zou X, Chen T, Xie G, Cheng Y, Dong T, Zhao L, Feng B, Hu X et al: Metabonomics identifies serum metabolite markers of colorectal cancer. Journal of Proteome Research 2013, 12(6):3000-3009.

24. Kim SY, Kim BK, Gwon MR, Seong SJ, Ohk B, Kang WY, Lee HW, Jung HY, Cho JH, Chung BH et al: Urinary metabolomic profiling for noninvasive diagnosis of acute $\mathrm{T}$ cell-mediated rejection after kidney transplantation. J Chromatogr B Analyt Technol Biomed Life Sci 2019, 1118-1119:157-163.

25. Zeng H, Tong R, Tong W, Yang Q, Qiu M, Xiong A, Sun S, Ding L, Zhang H, Yang L et al: Metabolic Biomarkers for Prognostic Prediction of Pre-diabetes: results from a longitudinal cohort study. Sci 
$\operatorname{Rep} 2017,7(1): 6575$.

26. Health UDo, Services H: Guidance for Industry, Bioanalytical Method Validation. http://wwwfdagov/cder/guidance/indexhtm 2001.

27. Han J, Qin WX, Li ZL, Xu AJ, Xing H, Wu H, Zhang H, Wang MD, Li C, Liang L et al: Tissue and serum metabolite profiling reveals potential biomarkers of human hepatocellular carcinoma. Clin Chim Acta 2019, 488:68-75.

28. Yang QJ, Zhao JR, Hao J, Li B, Huo Y, Han YL, Wan LL, Li J, Huang J, Lu J et al: Serum and urine metabolomics study reveals a distinct diagnostic model for cancer cachexia. J Cachexia Sarcopenia Muscle 2018, 9(1):71-85.

\section{Figures}



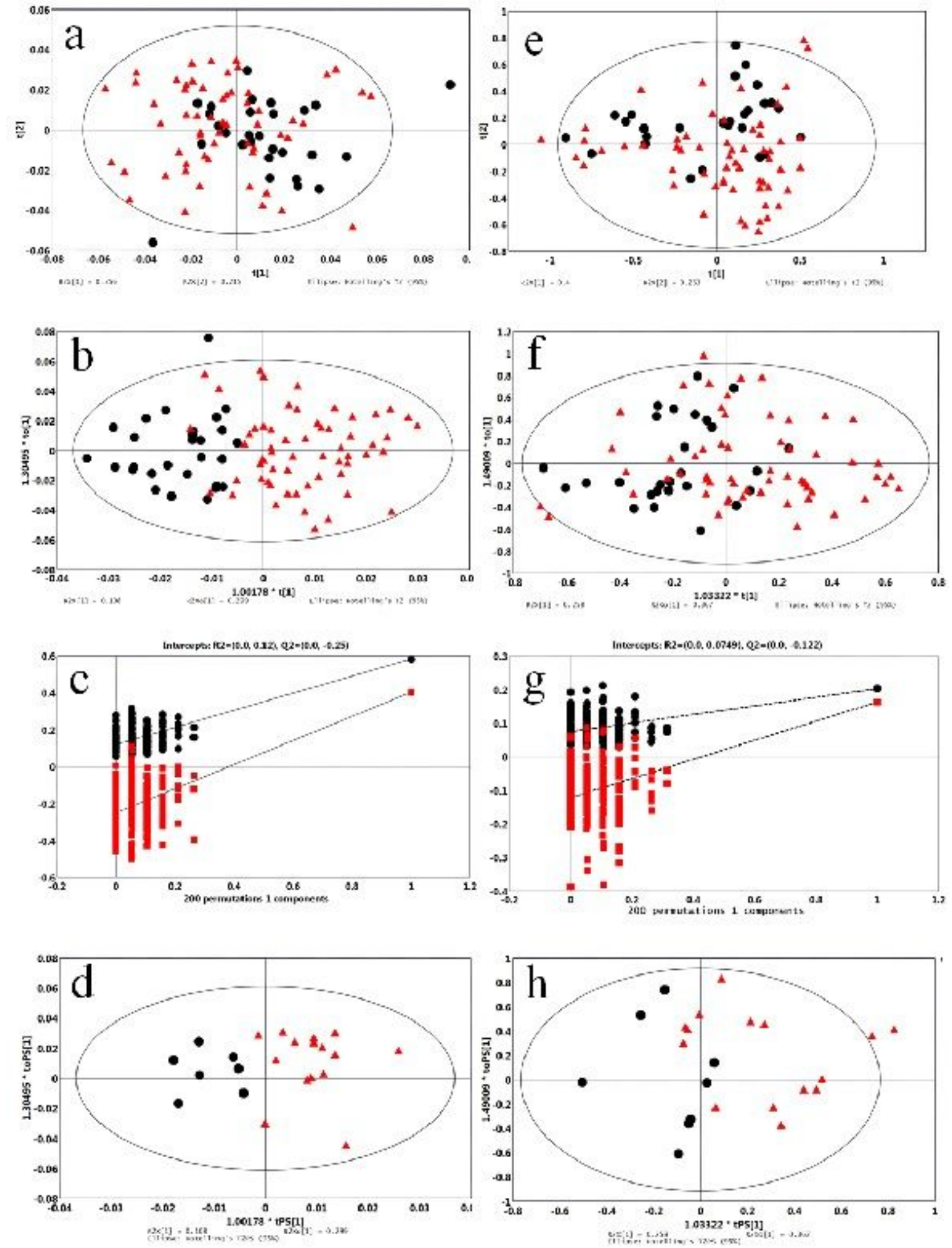

Figure 1

Overview of AA profiles of the USD (black) and HC (red) groups in serum and urinary samples, respectively. (a) PCA score plot, (b) OPLS-DA score plot and (c) validation plot with 100 permutation tests of the training set, (d) predicted OPLS-DA score plot of the test set of serum samples. (e) PCA score plot, (f) OPLS-DA score plot and (g) validation plot with 100 permutation tests of the training set, $(\mathrm{h})$ predicted OPLS-DA score plot of the test set of urinary samples. 


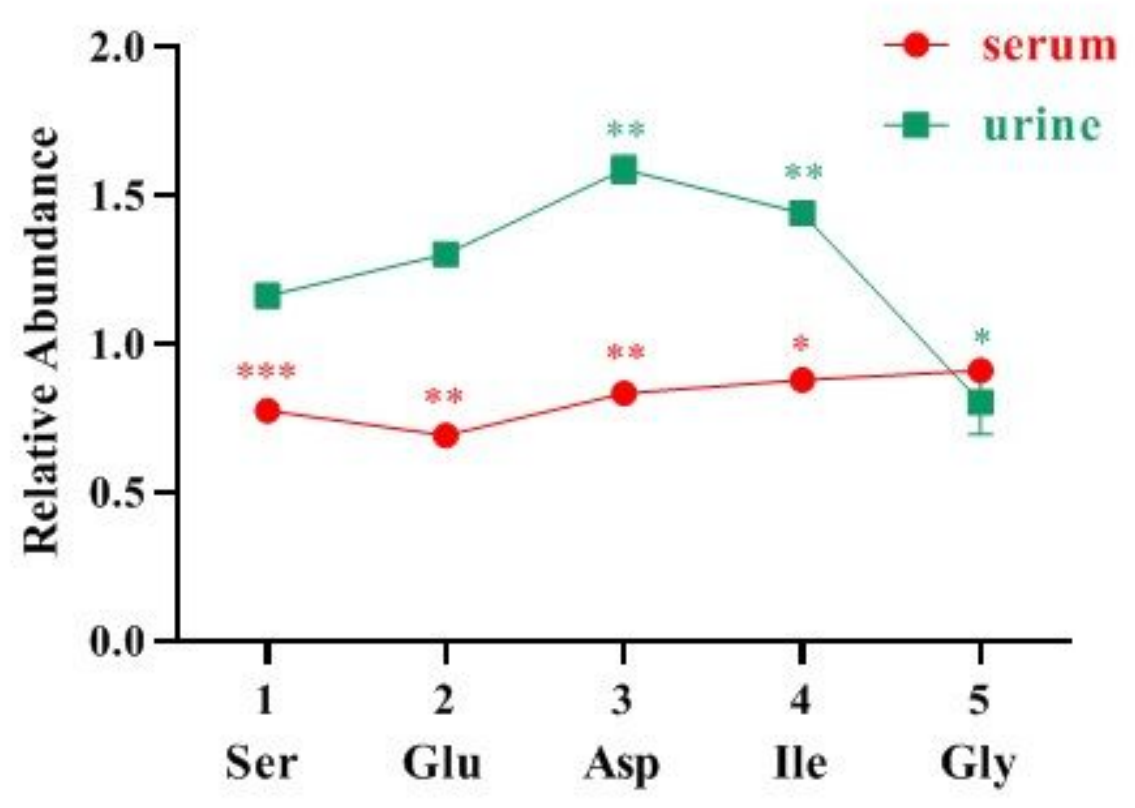

\section{Figure 2}

Fold changes (USD samples compared with HC samples) of Ser, Glu, Asp, lle and Gly in serum (red) and urinary (green) samples. ${ }^{*}, * \star, * \star \star$ represents for $p<0.05, p<0.01$ and $p<0.001$ in serum samples (red) and in urinary samples (green), respectively.

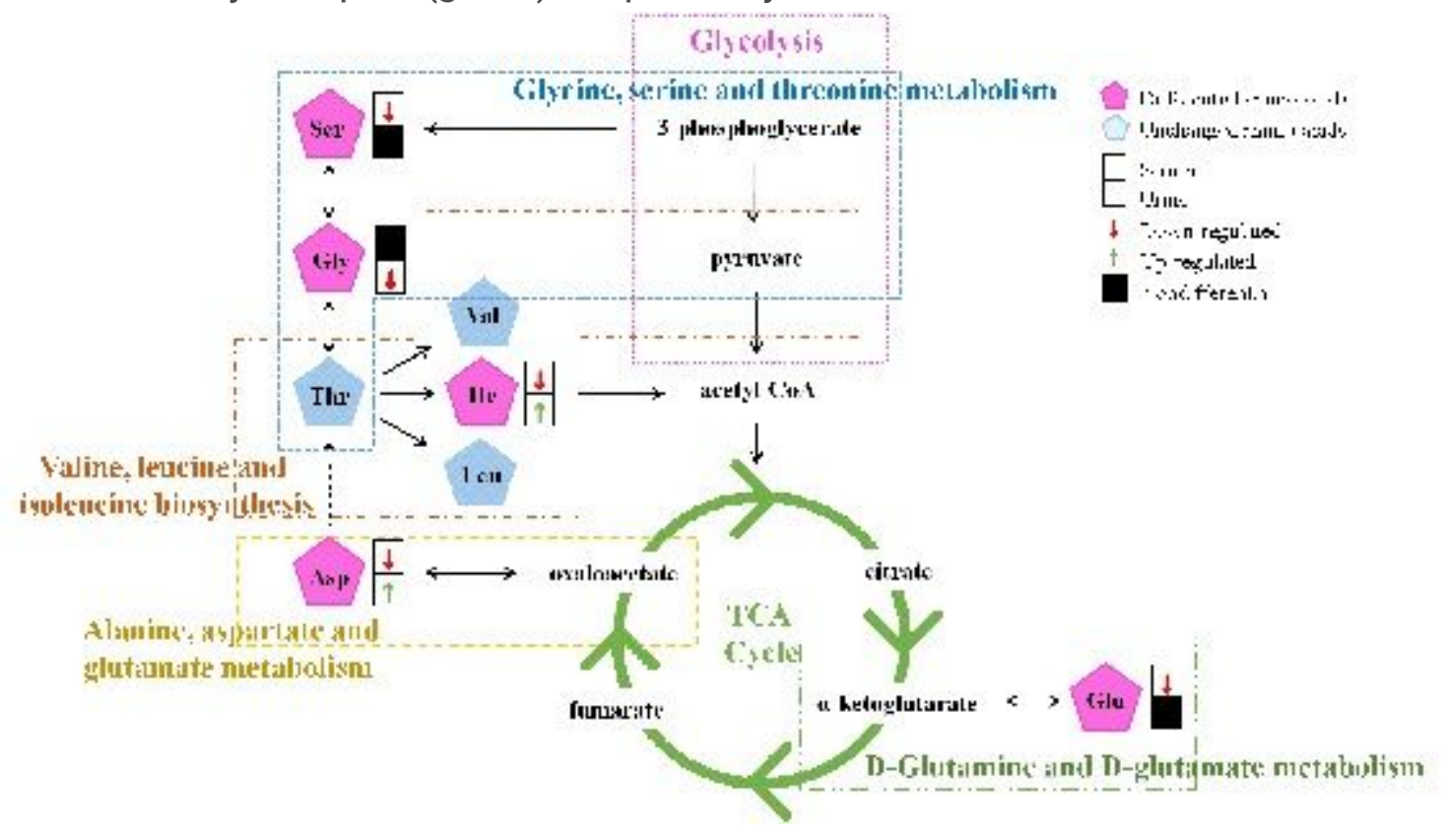

\section{Figure 3}

The metabolic networks of the USD-related altered AAs. 


\section{ROC Curve}

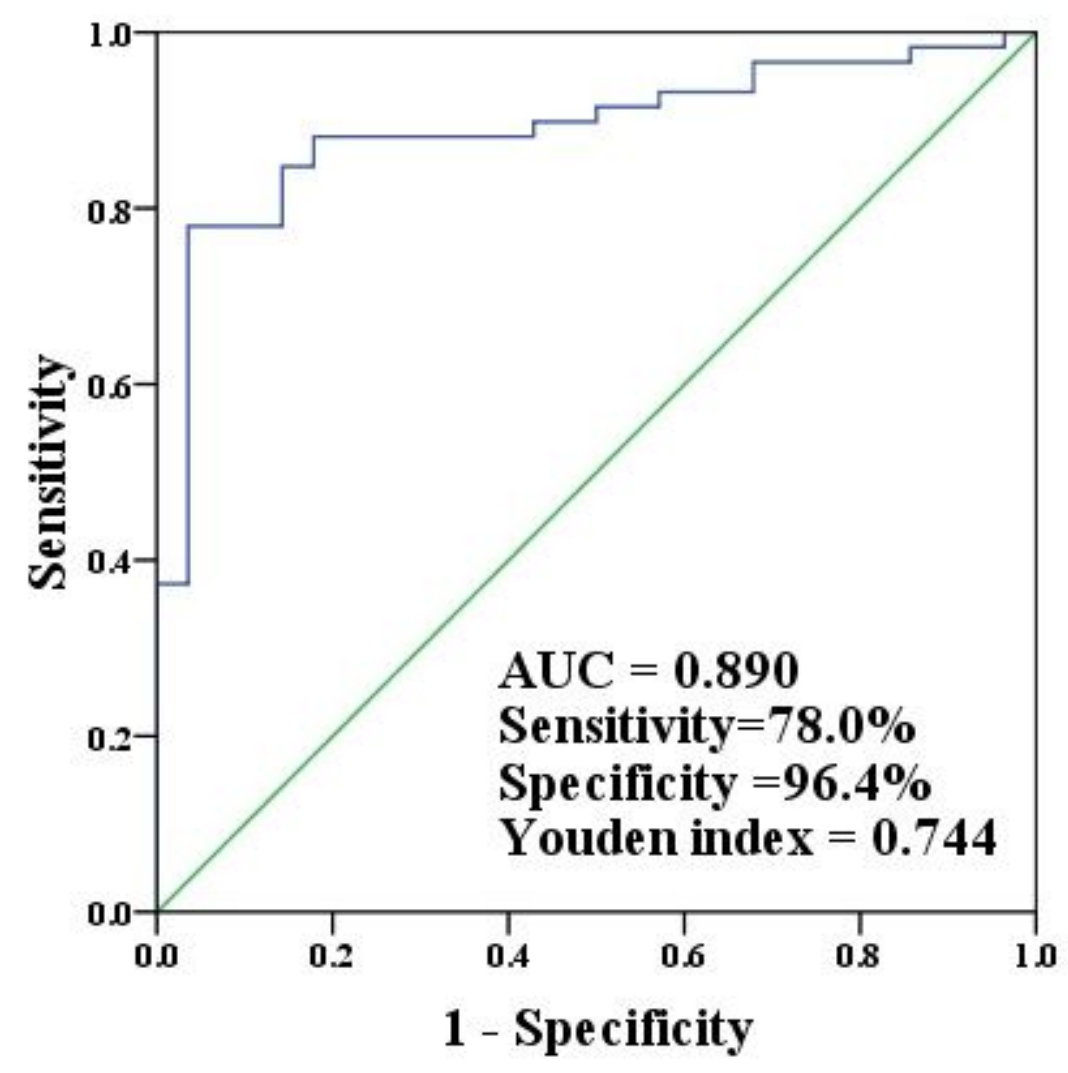

Figure 4

ROC curve of the combined panel of serum Ser, Glu, Asp, lle and urinary Gly and Asp by integrating.

\section{Supplementary Files}

This is a list of supplementary files associated with this preprint. Click to download.

- Supplementarymaterials.doc 